\title{
Bounds on vacuum-orthogonal Lorentz and $C P T$ violation from radiative corrections
}

\author{
Brett Altschul* \\ Department of Physics and Astronomy, University of South Carolina, Columbia, \\ South Carolina 29208, USA
}

(Received 30 April 2019; published 14 June 2019)

\begin{abstract}
Certain forms of Lorentz violation in the photon sector are difficult to bound directly, since they are "vacuum orthogonal"- - meaning they do not change the solutions of the equations of motion in vacuum. However, these very same terms have a unique tendency to contribute large radiative corrections to effects in other sectors. Making use of this, we set bounds on four previously unconstrained $d=5$ photon operators at the $10^{-25}-10^{-31} \mathrm{GeV}^{-1}$ levels.
\end{abstract}

DOI: 10.1103/PhysRevD.99.111701

Since the 1990s, there has been a great deal of renewed interest in the idea that some seemingly fundamental symmetries in physics, such as Lorentz and $C P T$ symmetries, might actually be very weakly violated in nature. Thus far, there is no compelling experimental evidence for such a conjecture. However, since these symmetries are perceived as being so basic that they underpin our current theories of elementary particle physics and of gravitation, it is worth understanding exactly how precisely the symmetries have been measured. If such putatively fundamental symmetries were found to be ever so slightly broken, that could change our expectations about the nature of physical laws at the most basic level. Here, we will introduce a new method for placing strong bounds on forms of Lorentz violation which have previously been considered quite difficult to observe.

There are a number of reasons why interest in searches for Lorentz and $C P T$ violation have picked up quite a bit in the last two decades. There has always been the motivation alluded to above - the notion that any principle that seems so fundamental ought to be studied and understood as precisely as possible. However, interest in broken Lorentz and $C P T$ symmetries expanded a great deal as it came to be realized that many of the conceptual frameworks that have been proposed as ways of describing quantum gravity seem to allow for Lorentz violation, at least in certain parameter regimes [1-6]. Moreover, it was also realized that older tests of Lorentz and CPT symmetry had not really done a good job of constraining the full parameter space of

\footnotetext{
*baltschu@physics.sc.edu
}

Published by the American Physical Society under the terms of the Creative Commons Attribution 4.0 International license. Further distribution of this work must maintain attribution to the author(s) and the published article's title, journal citation, and DOI. Funded by SCOAP ${ }^{3}$.
Lorentz and CPT violation. With the development of a systematic effective field theory (EFT) to describe the possible forms that Lorentz violation may take in the interactions of standard model fields, it became possible to make rigorous comparisons between the results of different types of Lorentz tests and to design new experiments to explore regions of the parameter space that have not previously been well constrained.

This EFT is known as the standard model extension (SME) $[7,8]$. Its action is built using standard model fields, constructed subject to the same requirements as regular standard model operators, except that they need not be invariant under rotations or Lorentz boosts. Since it is not possible to have a $C P T$-violating quantum field theory with a well-defined $S$-matrix without there also being Lorentz violation [9], the SME suffices as the most general EFT for describing $C P T$ violation as well as Lorentz violation. The SME, in its most general form, has an infinite tower of operators (of progressively increasing dimension) in its action, but when the additional requirement of renormalizability is imposed on the theory, the result is the minimal SME (mSME), which has a finite number of physically observable parameters. In many cases, it makes the most sense to parametrize the results of experimental Lorentz tests as bounds on linear combinations of mSME coupling parameters. However, there has also been growing interest in the higher-dimensional Lorentz-violating operators that are not part of the mSME-especially in the electromagnetic sector [10], where precise measurements of the polarization of light coming from cosmologically distant sources can often been used to place extremely stringent bounds. Depending on what assumptions are made, many of the same experiments that have been used to bound mSME parameters could also be interpreted as giving bounds on the coefficients of the nonminimal theory. A summary of the current bounds on many different SME coefficients may be found in [11]. 
However, there are some types of higher-dimensional photon Lorentz violation that cannot be bounded using birefringence measurements. In fact, some parameters are quite difficult to constrain by any technique. We shall provide new bounds on a class of nonminimal SME operators for photons - operators which are otherwise quite difficult to study. This will be accomplished through an examination of the quantum corrections that such operators can contribute to in other sectors of the SME that are easier to study.

The general form of a SME operator can be constructed as a product of standard model fields and their derivatives. However, unlike in the standard model, this product may have free Lorentz indices. These indices are then contracted with a constant tensor, which represents a preferred background in spacetime. The coefficients that make up this tensor are the quantities that experiments can be used to place bounds on. If Lorentz symmetry is broken spontaneously (as might be the case in a number of proposed quantum gravity frameworks), then the coefficient tensors are related to the vacuum expectation values of dynamical fields that possess tensor indices.

With increasing mass dimension, nonminimal SME operators get more and more derivatives, and each added derivative typically requires an additional index on the coefficient tensor, to be contracted with the index on $\partial_{\mu}$. The proliferation of indices means that the number of possible Lorentz-violating operators increases with the operator dimension. Moreover, in most cases, the presence of each additional derivative means an additional power of the energy in a term's observable effects on relativistic quanta. However, there are important exceptions; when two derivatives are contracted to form $\partial^{\mu} \partial_{\mu}=\partial^{2}$, the resulting term scales as the invariant mass squared of the quanta, not with the energy. This makes any term in the electromagnetic sector that includes a $\partial^{2}$ factor what is known as "vacuum orthogonal." The name comes from the fact that such terms are unobservable in the vacuum; any vacuum solution of the ordinary Maxwell's equations (with momentum $p^{2}=0$ in Fourier space), will also be a solution to the theory with the vacuum-orthogonal operator appended to it. This kind of behavior appears to make the vacuumorthogonal terms hard to constrain. For example, polarimetric measurements on the radiation from cosmologically distant sources has been tremendously useful in constraining the coefficients that lead to photon birefringence [11]; the resulting bounds can be extremely tight, because of the long propagation distances involved, but the technique cannot be used to constrain the vacuum-orthogonal terms. A completely different approach is needed for the vacuum-orthogonal sector.

Note that discrete symmetries $(C, P$, and $T)$ cannot be used to distinguish the vacuum-orthogonal operators containing $\partial^{2}$ from rotation-invariant operators involving $\partial_{0}^{2}$ or $\partial_{j} \partial_{j}$. Thus far, direct measurements of the vacuum-orthogonal terms have typically utilized matter-filled resonant cavities. The rest frame of the material breaks the boost invariance and ensures that the photons in the cavity will have $p^{2} \neq 0$.

Yet while the vacuum-orthogonal operators have few directly observable tree-level effects, they (unlike many other terms with $d>4$ ) are "unsafe" with respect to quantum corrections-in the sense that they can make direct (and large) contributions to the renormalization of lower-dimensional operators. Higher-dimensional operators with many Lorentz indices typically cannot make radiative contributions to $d=3$ and $d=4$ operators, because there are no $d \leq 4$ operators with the same Lorentz structures. A well known example of this phenomenon is that when a conventional gauge theory is regulated at short distances by a lattice, the low-energy behavior is Lorentz invariant, in spite of the use of a Lorentz-violating regulator. The Lorentz violation due to the lattice is irrelevant (in the renormalization group sense), because the dominant lattice effects are characterized by a symmetric four-index tensor, and there are no symmetric four-index tensor operators in the longdistance theory to "inherit" the Lorentz violation from the short-distance lattice.

However, when a Lorentz-violating operator of dimension $d$ includes a Lorentz scalar $\partial^{2}$ factor, the operator will have precisely the same tensor structure as another operator of dimension $d-2$. This means that the two terms may be intermixed by radiative corrections. We shall concentrate here on how a $d=5$ generalization of the Lorentz-violating Chern-Simons term in the photon sector contributes to a $d=3 \quad b$-type operator in the charged fermion sector. However, the phenomenon is fairly general. The radiative corrections involve virtual photons that are far off shell, with virtual momenta $p^{2} \sim \Lambda^{2}$, for some ultraviolet cutoff $\Lambda$. So for every factor of $\partial^{2}$ in the structure of a higherdimensional operator, there will be quantum corrections to lower-dimensional analogues that are enhanced by factors of the large quantity $\Lambda^{2}$.

The SME Lagrange density for a Lorentz-violating generalization of quantum electrodynamics (QED) with a nonminimal, vacuum-orthogonal, $d=5$ operator takes the form

$\mathcal{L}_{5}=-\frac{1}{4} F^{\mu \nu} F_{\mu \nu}+\frac{1}{2} \vec{k}_{5}^{\mu} \epsilon_{\mu \alpha \beta \gamma} F^{\alpha \beta} \partial^{2} A^{\gamma}+\bar{\psi}(i \not \partial-m-e \not A) \psi ;$

the $\neg$ atop the Lorentz violation coefficient tensor indicates its vacuum-orthogonal nature. The experimental significance of this operator is that it can make radiative corrections to a readily observable form of Lorentz violation in the fermion sector. This occurs through the insertion of a $\vec{k}_{5}$ vertex into the virtual photon propagator in the usual one-loop fermion self-energy diagram. 
The contribution this makes to the fermion self-energy is

$$
-i \Sigma(p)=-e^{2} \int \frac{d^{4} k}{(2 \pi)^{4}} \gamma^{\mu} S(k) \gamma^{\nu} D(p-k)\left[-i(p-k)^{2} \mathfrak{k}_{5}^{\alpha}(p-k)^{\beta} \epsilon_{\alpha \beta \mu \nu}\right] D(p-k),
$$

with $S(k)$ and $D(p-k)$ being the usual fermion and boson propagators. Inserting a Feynman parameter $x$ and shifting the integration variable to $\ell=k-x p$ gives

$$
-i \Sigma(p)=-2 e^{2} \epsilon_{\alpha \beta \mu \nu} \int_{0}^{1} d x x \int \frac{d^{4} \ell}{(2 \pi)^{4}} \frac{\gamma^{\mu}(\ell+x \not p+m) \gamma^{\nu} \hat{k}_{5}^{\alpha}[\ell-(1-x) p]^{2}[\ell-(1-x) p]^{\beta}}{\left(\ell^{2}-\Delta\right)^{3}},
$$

where $\Delta=(1-x) m^{2}-x(1-x) p^{2}$, as usual for the fermion self-energy. For on-shell fermions, $\Delta=(1-x)^{2} m^{2}$.

The largest radiative contribution will come from the $\mathcal{O}\left(\ell^{4}\right)$ term in the numerator. So the term from $-i \Sigma(p)$ with the greatest naive degree of divergence is

$$
-i \Sigma(p) \sim-2 e^{2} \epsilon_{\alpha \beta \mu \nu} \bar{k}_{5}^{\alpha} \int_{0}^{1} d x x \int \frac{d^{4} \ell}{(2 \pi)^{4}} \gamma^{\mu} \gamma_{\rho} \gamma^{\nu} g_{\sigma \tau} \frac{\ell^{\rho} \ell^{\sigma} \ell^{\tau} \ell^{\beta}}{\left(\ell^{2}-\Delta\right)^{3}} .
$$

The $\ell$-integration is quadratically divergent. Cutting off the integration with a regulator at a scale $\Lambda$ gives

$$
-i \Sigma(p) \sim-\frac{3 i e^{2}}{64 \pi^{2}} \Lambda^{2} \mathfrak{k}_{5}^{\alpha} \gamma_{\alpha} \gamma_{5} .
$$

This can be found, for example, using dimensional regularization and replacing the divergent $\Gamma$-function $\Gamma(1-d / 2) \rightarrow \Lambda^{2} / m^{2}$; the exact numerical coefficient will depend on the precise meaning of $\Lambda$ if a different regulator is used, but the result (5) will be sufficient for placing conservative, order-of-magnitude bounds on $\vec{k}_{5}$. This contribution to $\Sigma(p)$ is quite similar that made in a superficially renormalizable theory with a $d=3$ Chern-Simons term [12], differing only with the degree of the divergence.

The net result of this radiative correction is the addition of a term of the form $\bar{\psi} \not \gamma_{5} \psi$ to the effective Lagrange density for the fermions, where

$$
b^{\mu}=-\frac{3 e^{2} \Lambda^{2}}{64 \pi^{2}} \vec{k}_{5}^{\mu} .
$$

This would have immediate experimental consequences, since the $b$ coefficients give rise to a number of easily observed spin-dependent phenomena. Bounds on mSME coefficients such as $b$ are normally quoted in sun-centered celestial equatorial coordinates $(T, X, Y, Z)$. For two of the spatial components, $b_{X}$ and $b_{Y}$, the best bounds for a charged fermion species are for protons, made using a $\mathrm{He} / \mathrm{Xe}$ atomic magnetometer. The bounds are at the $\left|b_{X}\right|,\left|b_{Y}\right| \lesssim 10^{-32} \mathrm{GeV}$ level $[13,14]$. The best bounds on $b_{Z}$ (the component along the direction of the Earth's rotation) and the time component $b_{T}$ come from torsion pendulum measurements using magnetized samples containing macroscopic numbers of electron spins, with constraints at the $\left|b_{Z}\right| \lesssim 10^{-29} \mathrm{GeV}$ and $\left|b_{T}\right| \lesssim 10^{-26} \mathrm{GeV}$ levels $[15,16]$. (These two components of $b$ are harder to measure because it is not possible to just use the rotation of the Earth to search for their anisotropic effects.) Unfortunately, none of these bounds are "clean." All the constraints are on weighted sums of multiple SME coefficients, which are hard to disentangle in nonrelativistic experiments. However, if $k_{5}$ represents the ultimate source of all Lorentz violation in the theory, then there will be no induced $b$ for a neutral particle like a neutron, and the magnetometer and torsion pendulum bounds are just on the induced $b$ coefficient for the charged particles-which is universal by (6).

The extremely tight bounds on linear combinations containing the $b$ coefficients for charged fermions should translate into similarly stringent constraints on the $\vec{k}_{5}$ coefficients. However, to find useful numerical estimates, the issue of the quadratic divergence must be addressed. The SME, particularly when nonminimal terms are included, must be interpreted as an EFT; it is valid for calculations up to some scale, but a different ultraviolet completion of the theory is needed above that scale. Empirically, we know that QED is valid up to approximately the electroweak scale of $\sim 100 \mathrm{GeV}$. Nor is there any strong Lorentz violation up to this scale; this is known from collider searches for Lorentz violation with heavy particles such as the top quark $[17,18]$.

We shall therefore take $\Lambda \approx 100 \mathrm{GeV}$ as a lower estimate of the cutoff scale for the virtual momenta in the fermion self-energy. Based on the existing constraints on fermionic $b$ coefficients and setting bounds conservatively, at least an order of magnitude looser than given by direct application of (6), to allow for possible cancellations between the radiatively generated $b$ coefficients and those coefficients that are intrinsic to the fermion, the resulting constraints are

$$
\begin{gathered}
\left|\vec{k}_{5}^{X}\right|,\left|\vec{k}_{5}^{Y}\right| \lesssim 10^{-31} \mathrm{GeV}^{-1} \\
\left|\vec{k}_{5}^{Z}\right| \lesssim 10^{-28} \mathrm{GeV}^{-1}
\end{gathered}
$$




$$
\left|\vec{k}_{5}^{T}\right| \lesssim 10^{-25} \mathrm{GeV}^{-1} .
$$

Not only are these the first bounds placed on the coefficients for vacuum-orthogonal operators with $d=5$, they are not that dissimilar to many of the extremely tight bounds on other directly observable $d=5$ operators, placed using astrophysical birefringence measurements, which cover a $\sim 10^{-23}-10^{-34} \mathrm{GeV}^{-1}$ range [19-23].

This method of placing bounds is specific to the vacuumorthogonal terms. The reason that the operator parametrized by $\vec{k}_{5}$ is unsafe is that it has the same discrete symmetries and the same Lorentz structure as the fermion $b$ operators. Other bilinear electromagnetic terms with operator dimension $d=5$ will have the same number of derivatives, but without two of the derivatives being contracted to form a d'Alembertian; this requires the presence of additional indices on the coefficient tensor, and there are no observable $d=3$ operators with matching Lorentz structures.

Limits on forms of Lorentz violation that are not vacuum orthogonal typically scale as $E^{-(d-2)}$ or $E^{-(d-3)} L^{-1}$, where $E$ is the energy scale of the quanta involved in an experimental measurement and $L$ is the line of sight in an experiment measuring photons' polarizations or times of flight. For the vacuum-orthogonal terms in the action, the strength of any bounds based on radiative corrections to lower-dimensional operators involve the replacement of one factor of $E^{-2}$ by $\Lambda^{-2}$ for each factor of $\partial^{2}$. Since $\Lambda$ is, in principle, the scale up to which the SME is valid as a low-energy EFT, this can indicate significant improvement in the tightness of the bounds, relative to what may be possible for operators that are not vacuum orthogonal. This means that radiative corrections are the most natural source for strong bounds on the vacuumorthogonal Lorentz- and $C P T$-violating operators.

The radiative mixing of $\vec{k}_{5}$ and $b$ also works the other direction [24,25]; starting from an action containing a fermion $b$ term, quantum corrections will generate an infinite series of vacuum-orthogonal photon terms. Including all the terms at $\mathcal{O}(b)$, the effective Lagrange density due to $b$ insertions in the one-loop photon self-energy is

$$
\begin{aligned}
\Delta \mathcal{L}_{A F}= & \frac{e^{2}}{4 \pi^{2}} b_{\lambda} \epsilon^{\lambda \mu \nu \rho}\left\{\left[\frac{\sin ^{-1}\left(\sqrt{-\partial^{2}} / 2 m\right)}{\left(\sqrt{-\partial^{2}} / 2 m\right) \sqrt{1+\partial^{2} / 4 m^{2}}}-1\right] A_{\mu}\right\} \\
& \times\left(\partial_{\nu} A_{\rho}\right) .
\end{aligned}
$$

The transcendental function appearing in (10) can be expanded as a Maclaurin series, so long as $|\xi|<1$, where $\xi=\sqrt{-\partial^{2}} / 2 m=\sqrt{p^{2}} / 2 m$. (At $\xi=1$, there is the obvious branch cut, corresponding to the threshold for the creation of real fermion-antifermion pairs.) The first few terms of the series expansion are $\frac{\sin ^{-1} \xi}{\xi \sqrt{1-\xi^{2}}}-1=\frac{2 \xi^{2}}{3}+\frac{8 \xi^{4}}{15}+\frac{16 \xi^{6}}{35}+\frac{128 \xi^{8}}{315}+\cdots$

(The terms in this expansion suggest, but never quite achieve, a simple pattern.)

The subtraction of the $\mathcal{O}\left(\xi^{0}\right)$ term corresponds to having the $d=3$ Chern-Simons term vanish. The radiative corrections to this term were quite controversial at one point. In a pure Abelian gauge theory, not embedded in a larger theory that includes gravitation, and with explicit Lorentz violation in the form of a tree-level $b$ term, it turns out that the $d=3$ term is finite, yet also of undetermined magnitude. The ambiguity is related to the fact that the $d=3$ term with just one derivative is not, on its own, gauge invariant. (The integrated action is, however, gauge invariant, which is enough to ensure that the equations of motion are also invariant.) The ambiguity does not extend to the nonminimal terms, since they are fully gauge invariant, depending only on derivatives of $A$ rather than on $A$ itself.

Moreover, if the Abelian gauge theory is part of a larger theory that includes general relativity (or a more general metric theory of Riemannian gravity), matters are subtly different. Explicit breaking of Lorentz invariance by a $b$ term is not consistent with the metrical structure of spacetime [26]. Unless $b$ is actually derived from the vacuum expectation value of a separate axial vector field (endowed with its own nontrivial dynamics), the Bianchi identities that are required for the geometric interpretation of gravity cannot be satisfied. (There may be more general geometric theories of gravitation, perhaps utilizing Finsler geometry, that avoid this problem. However, these potential theories are, at present, too poorly developed to provide a framework for studying loop corrections involving quantum fields on these kinds of backgrounds.) The modified photon self-energy must be transverse to both of the external momenta, which are potentially different, since $b$, being a dynamical quantity, can carry momentum itself [27]. The presence of two independent transversality conditions forces the self-energy to be at least quadratic in momentum, which rules out the $d=3$ operator, but not the $d>4$ ones [28].

Unfortunately, the radiative corrections to $\vec{k}_{5}$ do not seem to be useful for setting any additional bounds. The coefficients $\vec{k}_{5}$ of the $d=5$, vacuum-orthogonal form of Lorentz violation are extremely difficult to constrain directly. What we have shown, however, is that $\vec{k}_{5}$ and similar terms are, on the other hand, uniquely susceptible to being constrained using radiative corrections. Existing bounds on the $b$ parameters for charged fermions can be interpreted as limits on the components of $\vec{k}_{5}$ at the $10^{-25}-10^{-31} \mathrm{GeV}^{-1}$ levels, not so different from the bounds on other $d=5$ operators which are not vacuum orthogonal. 
[1] V. A. Kostelecký and S. Samuel, Phys. Rev. D 39, 683 (1989).

[2] R. Gambini and J. Pullin, Phys. Rev. D 59, 124021 (1999).

[3] J. Alfaro, H. A. Morales-Técotl, and L. F. Urrutia, Phys. Rev. D 65, 103509 (2002).

[4] V. A. Kostelecký, R. Lehnert, and M. J. Perry, Phys. Rev. D 68, 123511 (2003).

[5] P. Hořava, Phys. Rev. D 79, 084008 (2009).

[6] J. W. Moffat, Classical Quantum Gravity 27, 135016 (2010).

[7] D. Colladay and V. A. Kostelecký, Phys. Rev. D 55, 6760 (1997).

[8] D. Colladay and V. A. Kostelecký, Phys. Rev. D 58, 116002 (1998).

[9] O. W. Greenberg, Phys. Rev. Lett. 89, 231602 (2002).

[10] V. A. Kostelecký and M. Mewes, Phys. Rev. D 80, 015020 (2009).

[11] V. A. Kostelecký and N. Russell, Rev. Mod. Phys. 83, 11 (2011); updated as arXiv:0801.0287v12.

[12] V. A. Kostelecký, C. D. Lane, and A. G. M. Pickering, Phys. Rev. D 65, 056006 (2002).

[13] F. Allmendinger, W. Heil, S. Karpuk, W. Kilian, A. Scharth, U. Schmidt, A. Schnabel, Yu. Sobolev, and K. Tullney, Phys. Rev. Lett. 112, 110801 (2014).

[14] Y. V. Stadnik and V. V. Flambaum, Eur. Phys. J. C 75, 110 (2015).
[15] B. R. Heckel, C. E. Cramer, T. S. Cook, E. G. Adelberger, S. Schlamminger, and U. Schmidt, Phys. Rev. Lett. 97, 021603 (2006).

[16] B. R. Heckel, E. G. Adelberger, C. E. Cramer, T. S. Cook, S. Schlamminger, and U. Schmidt, Phys. Rev. D 78, 092006 (2008).

[17] V. M. Abazov et al. (D0 Collaboration), Phys. Rev. Lett. 108, 261603 (2012).

[18] I. S. Karpikov, D. A. Tlisov, and D. V. Kirpichnikov, Phys. At. Nucl. 81, 257 (2018).

[19] F. W. Stecker, Astropart. Phys. 35, 95 (2011).

[20] K. Toma et al., Phys. Rev. Lett. 109, 241104 (2012).

[21] V. A. Kostelecký and M. Mewes, Phys. Rev. Lett. 110, 201601 (2013).

[22] F. Kislat and H. Krawczynski, Phys. Rev. D 95, 083013 (2017).

[23] A. S. Friedman, D. Leon, K. D. Crowley, D. Johnson, G. Teply, D. Tytler, B. G. Keating, and G. M. Cole, Phys. Rev. D 99, 035045 (2019).

[24] R. Jackiw and V. A. Kostelecký, Phys. Rev. Lett. 82, 3572 (1999).

[25] A. F. Ferrari, J. R. Nascimento, and A. Yu. Petrov, arXiv:1812.01702.

[26] V. A. Kostelecký, Phys. Rev. D 69, 105009 (2004).

[27] B. Altschul, arXiv:1903.10100.

[28] S. Coleman and S. L. Glashow, Phys. Rev. D 59, 116008 (1999). 